\title{
Condicionantes pedagógicas productoras de vulnerabilidad escolar en Educación Media Superior
}

\section{Pedagogic Conditioning factors that produce vulnerability in High School settings}

\author{
Eva América Mayagoitia Padilla \\ Universidad Pedagógica Nacional del Estado de Chihuahua \\ emayagoitia@upnech.edu.mx \\ Sandra Vega Villarreal \\ Universidad Pedagógica Nacional del Estado de Chihuahua \\ svega@upnech.edu.mx \\ Martha Carmela Talamantes Enríquez \\ Universidad Pedagógica Nacional del Estado de Chihuahua \\ mtalamantes@upnech.edu.mx
}

\section{Resumen}

La medición de la vulnerabilidad escolar en un subsistema de Educación Media Superior del Estado de Chihuahua, es objeto de este estudio diagnóstico, de carácter cuantitativo y descriptivo, cuyos resultados se obtienen de la aplicación de una encuesta valorada a través de una escala Likert, aplicada a una muestra estratificada de estudiantes. Su estudio permite explicar las causas que generan problemáticas de extraedad, rezago leve y grave y en muchos casos abandono definitivo de la escuela (Feijoo y Corbetta, 2004), implicando el análisis de factores extraescolares referidos a las condiciones personal y familiar, así como intraescolares que remiten a las condiciones institucional y pedagógica de las escuelas. Los resultados surgen del análisis de la dimensión pedagógica que involucró: las condiciones materiales de la clase; el dominio de contenidos por el profesor; las habilidades para facilitar el aprendizaje; el clima áulico; las dinámicas y relaciones de la clase; la evaluación del aprendizaje; la colaboración, apoyo escolar y el compromiso ético del profesor. Algunos de los riesgos encontrados refieren a los contenidos curriculares cuando se enfatiza que la mayoría de los maestros descuida una formación para la vida; la falta de impulso de los profesores a los procesos de aprendizaje y la utilización de la evaluación como instrumento de exclusión, pues pondera solamente la retención de contenidos, dejando de lado las necesidades de aprendizaje de los estudiantes, que no son otra cosa que el cumplimiento de sus derechos humanos fundamentales.

Palabras clave

Vulnerabilidad escolar, educación media superior, dimensión pedagógica. 


\begin{abstract}
The measurement of school vulnerability in a high school subsystem in the State of Chihuahua is the subject of this diagnostic study, of quantitative and descriptive nature, which results are obtained from the application of a survey assessed through a Likert scale, applied to a stratified sample of students. This study allows explaining the causes that generate the problems of over-age, slight and severe lag and in many cases definitive school abandonment (Feijoo and Corbetta, 2004), involving the analysis of extra-curricular factors regarding personal and family conditions, as well as intraschool; the institutional and pedagogical conditions of the schools. The results come from the analysis of the pedagogical dimension that involved: class' material conditions; the teacher's skills and knowledge; the skills to facilitate learning; the classroom environment; the dynamics and relationships of the class; the assessment of learning; collaboration, school support and the ethical commitment of the teacher. Some of the risks found refer to the curricular contents highlighting that the majority of teachers neglect a training for life; lacking participation in moving forward towards a learning processes and the use of the evaluation as an instrument of exclusion, only considering the retention of knowledge, leaving aside the learning needs of the students, which are nothing other than compliance with their fundamental human rights.
\end{abstract}

\title{
Keywords
}

School vulnerability, high school education, pedagogical dimension.

\section{Introducción}

En un contexto caracterizado por un conjunto de problemáticas entre las que destacan la desigualdad social, pobreza, hambre, explotación infantil, violencia, marginación y exclusión, entre otras; el cumplimiento del derecho universal a la educación, ocupa un papel prioritario en las políticas públicas de los estados nación, pues como lo reconoce Delors (1996), la educación encierra un tesoro, dado su carácter potenciador de todos los derechos humanos. Por ello los estudios relacionados con el cumplimiento de este derecho universal, cobran una importancia crucial en la actualidad.

Esta investigación tiene una relación directa con el tema y forma parte de un diagnóstico, que se está desarrollando con el propósito de evaluar el estado que guarda la vulnerabilidad escolar en las instituciones de Educación Media Superior en el Estado de Chihuahua, México, que es el nivel educativo donde actualmente se concentran los mayores retos educativos.
Particularmente, mide una de las cuatro dimensiones de la vulnerabilidad escolar, que se reconoce como un fenómeno complejo que produce que los niños (as) y jóvenes, se alejen paulatinamente de la escuela, desmantelando también, ciertos ritos personales y sociales que inciden en su desarrollo y en sus condiciones de vida (Terigi, 2007). La valoración de la vulnerabilidad escolar, implica así, adentrarse en el estudio de los factores extraescolares (dimensión personal y familiar) e intraescolares (dimensión institucional y pedagógica) de la enseñanza y el aprendizaje en la escuela, lo que permite explicar con mayores argumentos, las razones que se encuentran detrás de las problemáticas de extraedad, rezago leve y grave y en muchos casos el abandono definitivo de la escuela (Feijoo y Corbetta, 2004).

\section{Fundamentos teóricos}

Superando las tesis que reconocen a la vulnerabilidad como un problema inherente a 
los estudiantes y sus familias, se asume que se trata de un fenómeno complejo de carácter relativo e interactivo. Relativo porque involucra a situaciones objetivas y subjetivas que pueden aparecer o no en distintos momentos y etapas del proceso de escolarización. Interactivo porque tal como lo sostiene López (2004), es un fenómeno relacional que se juega entre lo que el alumno trae y lo que la escuela provee. Por ello, hablar de vulnerabilidad escolar, supone asumir una responsabilidad social que involucra al sistema educativo, a las autoridades, los padres de familia y también a los maestros, en la atención a esta problemática que pone en riesgo el futuro de millones de estudiantes.

En relación a la dimensión pedagógica, estudios realizados por Feijoó y Corbetta (2004), reconocen que el desempeño escolar suele ser predictor del que los estudiantes tendrán en otros órdenes institucionales. Se afirma así, que la escuela y el desempeño escolar son sin duda un logro, pero también, un pronóstico de la capacidad que tendrán los jóvenes para desenvolverse en otras esferas de la vida; de ahí que el estudio de esta dimensión resulta fundamental.

$\mathrm{Su}$ análisis incluye los indicadores siguientes: a) condiciones materiales de la clase, es decir, el equipamiento del aula y su constitución como un lugar donde se facilita aprender; b) dominio de contenidos por el profesor, así como las habilidades para facilitar su aprendizaje; c) clima en el aula, es decir, la organización, interacciones y comunicación en clase y su relación con el aprendizaje escolar; d) las dinámicas y materiales de la clase y su relación con el aprendizaje; e ) la evaluación del aprendizaje; f ) la colaboración y apoyo escolar de los profesores y su relación con el aprendizaje y g) el compromiso ético, entendido como la capacidad de los profesores de constituirse en ejemplos a seguir como personas $y$ profesionales.

\section{Método}

La investigación fue de corte eminentemente cuantitativo con un diseño no experimental, que no manipula la realidad, sino que la mide, para dar cuenta del estado que guarda la vulnerabilidad escolar. Dado su carácter diagnóstico tuvo un carácter descriptivo que no establece causalidad del fenómeno estudiado.

La población se conformó por 2572 estudiantes inscritos durante el ciclo escolar 2015 - 2016 en uno de los subsistemas de Educación Media Superior. Se estimó una muestra probabilística estratificada, que por su naturaleza permitió que todos los estudiantes tuvieran la misma posibilidad de ser elegidos, a la vez que permitió determinar los niveles de error. La medición de la vulnerabilidad escolar en su dimensión pedagógica, se realizó a través de un instrumento tipo encuesta con base en escala Likert, para su aplicación a los estudiantes, lo que permitió medir la intensidad de cada uno de los indicadores que la componen. Para su validación se utilizó el método de test y retest, con la prueba estadística $\mathrm{R}$ de Pearson, obteniéndose una confiabilidad altamente positiva.

\section{Resultados}

El análisis descriptivo de los datos evidenció la presencia de diversas condicionantes que inciden en que el estudiante sea proclive a reprobar, suspender su trayectoria académica o incluso, abandonar la escuela. Entre ellos destacaron los relacionados con la relevancia de los contenidos abordados por el profesor, la organización y manejo de la clase, la confianza que genera en los estudiantes y el apoyo que les brinda como apoyo a su aprendizaje.

La primera interrogante valoró la pertinencia y utilidad de los contenidos trabajados en clase, tanto para sus estudios actuales, como para la carrera que visualizan a futuro. Las respuestas mostraron que la 
mayoría los considera altamente útiles y pertinentes, dado que sólo el 11 \% les restó valor. Se concluye así, que el currículo que se ofrece a los estudiantes no es en sí mismo una causante de fracaso escolar. Sin embargo, resulta necesario cuestionar porque se valora su pertinencia, a la vez que se asumen con dificultad y disgusto.

En relación al dominio de los contenidos curriculares, el mismo $89 \%$ de estudiantes, consideró que los maestros conocen lo que enseñan y realizan un manejo adecuado de los temas, lo que facilita su aprendizaje en clase. Tales resultados ratifican el hecho de que el curriculum no aparece como un factor de riesgo en las trayectorias académicas de los estudiantes.

Otra pregunta indagó la forma en que los profesores organizan y desarrollan la clase. Los resultados mostraron que casi la totalidad de los encuestados, perciben que existe muy buena organización de la clase y que las dinámicas que en ella se desarrollan son pertinentes, adecuadas, lo que les facilita aprender los contenidos. De allí que este aspecto central del trabajo docente, tampoco es considerado como un factor de riesgo para la mayoría de los estudiantes.

Al cuestionar sobre los apoyos que se les brindan - niegan para resolver dudas, problemas o necesidades de aprendizaje, más de la mitad concuerda en que los profesores están dispuestos a acompañarles y que les facilitan, la mayoría de las veces, los recursos que necesitan para adquirir competencias disciplinares de la materia que imparten, lo que contrasta con un porcentaje similar que sostiene que no recibe apoyo. Alternamente, reconocen que no cuentan con apoyos para el desarrollo de competencias socioemocionales, que trasciendan el currículo disciplinar y preparan a los estudiantes para la construcción un proyecto profesional y personal basado en la acción positiva frente a los dilemas de la vida en sociedad.

El acto pedagógico contiene en sí mismo una amplia gama de implicaciones en la formación de ciudadanos y sociedades; de ahí que resultó necesario interrogar sobre el tipo de relación que los estudiantes establecen con sus maestros durante el proceso de aprendizaje. La conclusión general es que están satisfechos al sostener que se genera una relación cercana, de confianza, apoyo y solidaridad. Alternamente, una minoría reconoce la falta de impulso de los profesores a su proceso de aprendizaje, por lo que este factor constituye un elemento que no se debe descuidar en ningún momento, si se quiere impulsar una pedagogía inclusiva que reduzca los riesgos que vulneran las trayectorias estudiantiles.

Reconociendo que en las sociedades neoliberales, se ha instituido a la evaluación como un instrumento de exclusión, porque a partir del establecimiento de estándares de desempeño, se vulnera a quienes no reúnen las condiciones para alcanzarlos; se indagó sobre esta herramienta pedagógica, que utilizada únicamente como medición de resultados, puede poner en riesgo la estadía de los estudiantes en las escuelas.

La valoración de los estudiantes respecto a los criterios e instrumentos que se utilizan para evaluar los aprendizajes esperados, evidenció que un poco más de la mitad considera que son adecuados a los contenidos tratados en clase. Vale la pena recuperar, sin embargo, las opiniones de un sector, cercano al $50 \%$ de la población, que considera a estos elementos, como un factor que incide negativamente en su permanencia en la escuela, dado que los criterios utilizados para valorar su desempeño, no se convierten en pautas para la mejora del desempeño. Incluso algunos consideran injustas las calificaciones que los profesores les asignan, pues sienten que la evaluación no recupera su 
esfuerzo y dedicación; de ahí que resulte necesario profundizar en el estudio de este factor, como elemento productor de vulnerabilidad escolar.

\section{Conclusiones}

La vulnerabilidad escolar es un fenómeno complejo, cuyo incremento en la actualidad, obliga a la necesidad de situarlo como una prioridad en el diseño de políticas públicas, contrarrestando las tendencias mundiales que descuidan e incluso niegan sus implicaciones en las trayectorias escolares y en el logro educativo de los estudiantes.

Sin desconocer la importancia de las cuatro dimensiones que conforman el fenómeno (personal, familiar, institucional y pedagógica), en este estudio se priorizó el análisis de la dimensión pedagógica, a fin de develar sus implicaciones en la presencia ausencia de este fenómeno, lo que deriva en el reconocimiento de la responsabilidad docente en este fenómeno causante del rezago y abandono escolar en la educación media

Los resultados, mostraron que existen algunos factores que no son considerados como elementos desfavorables para la permanencia escolar, entre ellos los contenidos curriculares que son considerados como útiles y pertinentes, para los estudios actuales y para la profesión futura, lo que coincide con las opiniones mayoritarias sobre el dominio y manejo de los contenidos por parte de los profesores .

Contrariamente, aunque la mitad de los estudiantes sostiene que los profesores les apoyan cuando tienen dudas sobre los contenidos curriculares, otro porcentaje similar plantea que no los recibe. Alternamente queda en evidencia la atención disciplinar que caracteriza a este nivel educativo cuando se enfatiza que la mayoría de los maestros descuida el desarrollo de competencias socioemocionales características de una formación para la vida, inserta en una realidad compleja que traspasa los linderos del salón de clase y que reconoce que los alumnos son seres sociales inmersos en un proceso de humanización, que trasciende a las concepciones de formación como preparación para el desempeño de un puesto de trabajo o de una profesión.

Otro indicador de vulnerabilidad fue la evaluación, que es utilizada como instrumento de exclusión, dado que los criterios e instrumentos que se utilizan no contribuyen en la mejora de los aprendizajes, lo que demanda situarla al servicio de procesos académicos que garanticen una trayectoria escolar sin riesgos.

En síntesis, se sostiene que aunque los resultados no resultaron alarmantes, evidencian la necesidad de mejorar los procesos evaluativos, así como los mecanismos de apoyo a los estudiantes en riesgo, al igual que la formación en competencias socioemocionales, como una necesidad urgente en la formación de ciudadanos y de sociedades más humanas y menos excluyentes.

\section{Referencias}

Delors, J y otros. (1996). La educación encierra un tesoro. Madrid: Santillana, UNESCO

Fruggeri, L. (2001). Los conceptos de mononuclearidad y plurinuclearidad en la definición de familia. Revista del Centro Milanese di Terapia de lla Famiglia. Connessioni, 8. (Traducción de Felipe Gálvez Sánchez). Recuperado de:

win.associazioneculturaleepisteme.co $\mathrm{m} / \ldots /$ Mononuclearidad $\% 20 \mathrm{y} \% 20 \mathrm{pl} . .$.

López, (2004). Educación y Equidad. Algunos aportes desde la noción de educabilidad. Buenos Aires: Instituto Internacional de Planeamiento de la Educación/ UNESCO. Disponible en: http://unesdoc.unesco.org/images/00 14/001417/141736s.pdf.

Navarro, L. (2004). La escuela y las condiciones sociales para aprender y 
enseñar. Equidad social y educación en sectores de pobreza urbana. Chile: IPEUNESCO. Sede Regional Buenos Aires.
Terigi, F. (2007). Las trayectorias escolares. Del problema individual al desafío de política educativa. Buenos Aires. OEA 